\title{
Towards the Ultimate Membranes: Two- dimensional Nanoporous Materials and Films
}

\author{
Kumar Varoon Agrawal ${ }^{*}$
}

\begin{abstract}
The energy-efficient separation of molecules has been a popular topic in chemistry and chemical engineering as a consequence of the large energy-footprint of separation processes in the chemical industry. The Laboratory of Advanced Separations (LAS) at EPFL, led by Prof. Kumar Varoon Agrawal, is focused to develop next-generation, high-performance membranes that can improve the energy efficiency of hydrogen purification, carbon capture, hydrocarbon and water purification. For this, LAS is seeking to develop the ultimate nanoporous membranes, those with a thickness of $1 \mathrm{~nm}$ and possessing an array of size-selective nanopores. In this article, the research activities at LAS, especially in the bottom-up and top-down synthesis of chemically and thermally stable, nanoporous two-dimensional materials and membranes are discussed.
\end{abstract}

Keywords: Membranes · Metal-organic frameworks · Nanoporous graphene · Two-dimensional materials · Zeolite nanosheets

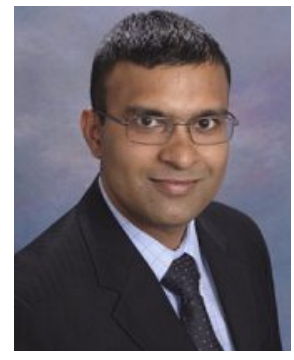

Prof. Kumar Varoon Agrawal is a tenure-track Assistant Professor leading the Laboratory of Advanced Separations (LAS) at the Institute of Chemical Sciences and Engineering at EPFL. He received his undergraduate degree in Chemical Engineering from IIT Bombay in 2005. Following this, he worked on product design at the global R\&D division of Procter \& Gamble, Kobe, Japan. In 2008, he joined the University of Minnesota to pursue a $\mathrm{PhD}$ in Chemical Engineering. The thesis work, advised by Prof. Michael Tsapatsis and Prof. Lorraine Francis, on the synthesis of two-dimensional zeolite

${ }^{*}$ Correspondence: Prof. K. V. Agrawal

École Polytechnique Fédérale de Lausanne (EPFL) Institute of Chemical Sciences and Engineering (ISIC) EPFL Valais Wallis

Rue de l'Industrie 17

Case Postale 440

$\mathrm{CH}-1950$ Sion

E-mail: kumar.agrawal@epfl.ch nanosheets and related membranes opened a new field of zeolite nanosheet-based high-performance membranes (Science 2011, 334, 72). In 2014, he joined the research group of Prof. Michael Strano at the Massachusetts Institute of Technology as a postdoctoral associate. His study of the phase transition of water confined inside isolated carbon nanotubes revealed exotic freezing transition behavior with water maintaining its frozen state up to $138{ }^{\circ} \mathrm{C}$ (Nature Nanotechnology 2017, 12, 267). Prof. Agrawal is the recipient of several awards including the IIT Bombay Institute Silver Medal (2005), Manudhane Best Undergraduate Student award (2005), University of Minnesota Doctoral Degree Fellowship (2012), Sigma Xi Award (2013), AIChE Separations Division Graduate Student Research Award (2013), and NAMS Young Membrane Scientist Award (2018).

\section{Challenges and Opportunities in the Separation Processes}

Separation processes are omnipresent in the chemical and the pharmaceutical industries attributing to the necessity to purify raw materials (removal of impurities, solvents, isotopes, etc.) and products (recycling of reactants and removal of byproducts from side-reactions, chain-reactions, etc.). The energy footprint of the separation processes can reach $40-70 \%$ of the total energy footprint of a chemical plant. [1] Chemical industries in most developed countries account for $20-40 \%$ of the total energy consumption, and therefore, the separation processes have a large global energy footprint. For example, separation processes in the United States (US) account for $c a .16 \%$ of its entire energy consumption. ${ }^{[2]}$ From the energy-footprint point of view, luckily, there are several avenues that can improve the energy-efficiency of separation processes. For instance, processes requiring extensive thermal energy (distillation of closely boiling molecules) can be modified using heat-integration. Alternatively, they can be partially or completely replaced by processes that demand lower energy. Process efficiency can be also improved by synthesizing high-performance materials that improve the heat and the mass transfer. Let's consider the case of water purification. Reverse-osmosis (RO) membranes are commonly used to purify water from contaminated resources (household and industrial wastewater) and seawater as a result of the ever-increasing scarcity of clean drinking water. RO can be an expensive process where the osmotic pressure of the solution is high. For instance, the osmotic pressure of seawater with $33 \mathrm{~g} / \mathrm{L}$ of $\mathrm{NaCl}$ is 27.8 bar, requiring a minimum of $2.82 \mathrm{~kJ} /$ liter of energy to desalinate. Here, the development of a membrane with a high salt rejection, approaching $100 \%$, would be highly attractive because this will allow a single-stage membrane operation where the seawater stream would be compressed only once. On the chemistry front, the challenge is to synthesize a membrane material that allows for near $100 \%$ rejection and that is resistant to the fouling and chlorinated water. Another extremely crucial separation is the capture of $\mathrm{CO}_{2}$. The $\mathrm{CO}_{2}$ concentration 
in the atmosphere has continuously risen in the last century to unprecedented levels, reaching 408.5 parts per million (ppm) in January 2018. This has severe consequences in the global temperature rise. For example, 2016 was the warmest year on record. The largest difficulty in cutting $\mathrm{CO}_{2}$ emission is that renewable energy sources (solar, wind, etc.) are still years away from replacing fossil fuels, and the implementation of carbon capture and sequestration increases the overall cost of the process significantly. A widely-implemented technology for $\mathrm{CO}_{2}$ capture is amine-based absorption where $\mathrm{CO}_{2}$ is chemisorbed in amines to form carbamates. However, the environmental friendliness and the energy-efficiency of the process, especially in decentralized operations, is questionable. Here, synthesis of i) porous adsorbent with a high working capacity and a low regeneration energy, ${ }^{[3]}$ and ii) $\mathrm{CO}_{2}$ selective highpermeance ( $>3000$ gas permeance units, GPU) membranes is highly attractive. ${ }^{[4]}$ Permeance is defined as gas flux normalized by the transmembrane pressure difference, and $1 \mathrm{GPU}$ is equivalent to $3.3 \times 10^{-10}$ mole $\mathrm{m}^{-2} \mathrm{~s}^{-1} \mathrm{~Pa}^{-1}$.

There are several other separation processes where the energy-efficiency can be improved. Examples include separation of benzene derivatives, alkenes from alkanes, organic solvents from solutes, decentralized (small-to-medium-scale) air separation (into $\mathrm{O}_{2}$ and $\mathrm{N}_{2}$ ), etc. Currently, air purification is carried out by cryogenic distillation which is energy-efficient on the large-scale but is quite expensive to decentralize or develop into a portable application. Here, a membrane-based $\mathrm{O}_{2} / \mathrm{N}_{2}$ separation could be a solution, however, the $\mathrm{O}_{2}$ permeance of the current polymeric membranes is too low, which increases the overall cost of separation. ${ }^{5]}$ Petrochemicals (benzene derivatives, olefins, paraffins) are primarily separated by thermal-driven processes such as distillation, crystallization, etc. For example, separation of $p$ xylene from a mixture of xylene isomers, produced in the toluene disproportionation reaction, is highly energy-intensive. The difference in the boiling point of $p$ - and $o$-xylene is only $5.6^{\circ} \mathrm{C}$, and their separation by distillation requires a large number of equilibrium stages, and a high reboiler duty. Emerging membrane technologies for either pre-concentrating the xylene feed for subsequent distillation ${ }^{[6]}$ or for effectively separating $p$-xylene ${ }^{[7]}$ is expected to cut down the overall cost of separation.

\section{Advantages of Membrane-based Separation}

A membrane-based process provides novel energy-efficiency opportunities both as a standalone separation unit and when combined with other processes. Membrane-based processes are one of the simplest unit operations requiring a low maintenance. Membranes are conducive to miniaturization and decentralization, and can be applied in portable applications (fuel cell membranes, portable oxygen purifiers, etc.). Recent developments in material chemistry, coating and crystallization techniques, and process design have made the prospect of membrane-based separation highly promising. Membrane separation relies on the chemical potential gradient across a thin film which is created by pressurizing the molecular mixture on the feed side, and in some cases, by applying a partial vacuum on the permeate side. Overall, the energy expenditure is primarily incurred due to compression and aspiration, and rarely on heating/cooling. As a result, membrane processes become attractive when the mixture feed is already available at a high pressure (for example, natural gas from the gas wells or products from a high-pressure reactor). Membranes can also be energy-efficient for separation of streams at low pressure (1 bar) where the mole fraction of the permeable molecule is 0.15 or higher (for example, postcombustion $\mathrm{CO}_{2}$ capture). ${ }^{[4,8]} \mathrm{A}$ distinct advantage of membrane processes is that they can be interfaced with the chemical reactor leading to process-intensification. For equilibrium-limited reactions, such as the water-gas shift reaction, the implementation of membrane reactor has been shown to increase the overall conversion. ${ }^{[9]}$ Further, a subset of membranes (inorganic membranes) can directly purify streams from a high-temperature reactor without requiring the stream to be cooled down.

\section{Emerging Membrane Platform}

Polymeric membranes have dominated the membrane separation field due to the ease of processing polymers into thin selective films. Typically, the polymeric membranes are prepared by casting or spinning a polymer solution in a flat sheet or a hollow-fiber morphology. As a result, a large membrane area (several hundred $\mathrm{m}^{2}$ ) can be prepared in a single day. However, there are several challenges with the polymeric membranes which limit their application. One of the biggest issues is a tradeoff in their separation performance (separation selectivity vs. permeability; permeability is defined as permeance normalized with the thickness of the selective layer). ${ }^{[10]}$ For membrane separation, a combination of high separation selectivity and high permeance is desired so that a single-stage membrane process can be applied with a relatively small membrane area. Because of the intrinsic tradeoff, highly permeable polymers yield a low separation selectivity. The origin of the tradeoff lies in the transport mechanism of molecules across the polymer film. The sorption-diffusion mechanism relies on the free-volume (which determines solubility of molecules in the polymer film) and the chain stiffness (which determines activation energy for the molecular diffusion). Stiffer chains usually lead to better selectivity but also increase activation energy for diffusion and therefore yield lower permeability. Other issues with the polymeric membranes include their poor chemical (plasticization, degradation, etc.), thermal (difficult to integrate with high temperature reactors) and mechanical stability (limits the thickness of the skin-layer). Recent progress in polymer chemistry have led to improved separation performances, especially with the development of thermally reduced (TR) polymers, ${ }^{[11]}$ and polymers with intrinsic microporosity (PIMs). ${ }^{[12]}$

The Laboratory of Advanced Separations (LAS) is engaged in synthesizing ultimate membranes that significantly exceed the performance limit of polymeric membranes, making the separation processes for gases and vapors highly energy-efficient. Since the membrane separation process is usually diffusioncontrolled, the synthesis of the ultimate membranes amounts to synthesizing the thinnest-possible molecular-selective barriers that are stable in the operating conditions. Naturally, two-dimensional (2D) films hosting molecular-selective pores are the ultimate membranes because the flux through the membrane is inversely proportional to the membrane thickness (Figs. 1A, B, and Eqn. (1)).

$$
J=D H \frac{\left(C_{\text {feed }}-C_{\text {permeate }}\right)}{l}
$$

where $J$ is the diffusive flux of molecules across the film, $D$ and $H$ are the diffusion and the adsorption coefficients, respectively, for the permeating molecules. $C_{\text {feed }}$ and $C_{\text {permeate }}$ are bulk phase concentration of permeating molecules, and $l$ is the membrane thickness. For a strictly two-dimensional nanoporous film, the pore-size and the mean free path of the gas/vapor molecules would determine the transport mechanism. If the pore diameter, $d_{\text {pore }}$ is larger than the mean free path of the molecule, $\lambda_{\text {mol }}$, the viscous flow, described by the modified Sampson's formula ${ }^{[13,14]}$ dominates (Fig. 1C, Eqn. (2)):

$$
J=\frac{d_{\text {pore }}\left(1+0.894 \kappa^{1.5}\right)}{6 \pi \mu} \Delta P
$$

where $\kappa$ is the porosity, $\Delta P$ is the transmembrane pressure difference and $\mu$ is the 
gas viscosity. With smaller pore diameters such that $d_{\text {mol }}<d_{\text {pore }}<\lambda_{\text {mol }}$, where $d_{\text {mol }}$ is the kinetic diameter of the molecule, effusive flux dominates as described by Eqn. (3):

$$
J=\frac{1}{\sqrt{2 \pi M R T}} \kappa \Delta P
$$

where $M$ is the molecular weight of the molecule, $R$ is the universal gas constant, and $T$ is the temperature. When the pore diameter is comparable to the kinetic diameter of the translocating molecule, $d_{p o r}$ $\approx d_{\text {mol }}$, activated transport dominates as described by Eqn. (4):

$$
\begin{aligned}
& J=C_{o} A_{a} A_{H} \exp \left(-\frac{E_{a}+\Delta H}{R T}\right) \\
& \left(\frac{P_{F}}{1+A_{H} \exp \left(-\frac{\Delta H}{R T}\right) P_{F}}-\frac{P_{P}}{1+A_{H} \exp \left(-\frac{\Delta H}{R T}\right) P_{P}}\right)
\end{aligned}
$$

where $C_{0}$ is the pore-density, $E_{\mathrm{a}}$ is the activation energy for the pore translocation, $\Delta H$ is the molecular adsorption energy. $A_{\text {a }}$ and $A_{\mathrm{H}}$ are pre-exponential factors corresponding to translocation and adsorption, respectively. $P_{\mathrm{F}}$ and $P_{\mathrm{p}}$ are the gas partial pressures on the feed and the permeate sides, respectively.

In contrast to the polymeric membranes where the polymeric chains have to undergo conformational changes for sorption-diffusion, the inorganic nanopores of
2D materials are relatively rigid and yield a high molecular diffusivity. Therefore, a high permeance can be achieved with the inorganic nanoporous 2D membranes. The mechanical strength of the inorganic nanoporous films is sufficiently high to allow fabrication of extremely thin films down to the thickness of a single-atom as in the case of graphene. To realize these 2D membranes, LAS has established a multidisciplinary program in chemistry, material science and chemical engineering, focusing on the synthesis, processing, and characterization of inorganic, nanoporous 2D materials (single-layer graphene, nanosheets derived from layered nanoporous materials). LAS is studying several synthetic routes (chemical vapor deposition (CVD), molecular layer deposition (MLD), hydrothermal and solvothermal synthesis, etc.), crystallization mechanism (induction time, nucleation density, anisotropic growth rates, substrate effects, grain-boundaries, etc.), post-synthetic modification including surface functionalization, ion-exchange, and exfoliation (Fig. 2). On the theoretical front, an improved understanding of structure-property relationships is being investigated by $a b$ initio molecular modeling (density functional theory), as well as by modeling adsorption and diffusion across the nanoporous 2D film. Overall, LAS is developing three distinct membrane platforms, a)

A

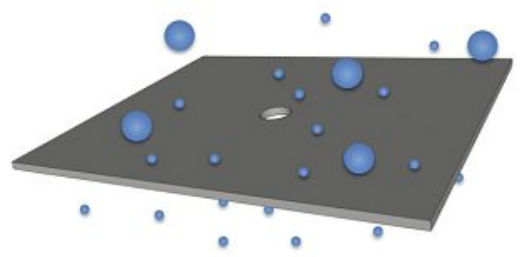

B

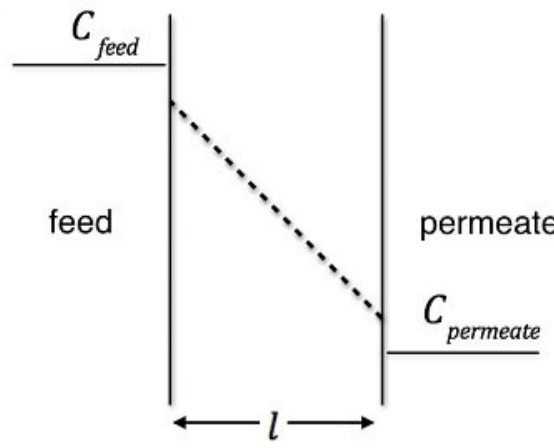

C

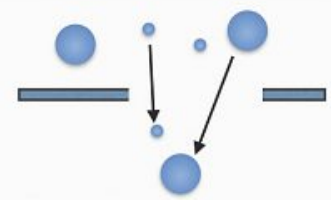

Gas phase transport

Viscous flow ( $\left.d_{\text {pore }}>\lambda_{\text {mol }}\right)$

Effusion $\left(d_{\text {mol }}>d_{\text {pore }}>\lambda_{\text {mol }}\right)$ single-layer nanoporous graphene films, b) ultrathin polycrystalline MOF films, and c) exfoliated nanoporous 2D nanosheets. These are discussed in detail below.

\subsection{Single-layer Nanoporous Graphene Membranes}

Graphene is a crystalline, 2D array of $\mathrm{sp}^{2}$-hybridized carbon, found in nature in its stacked form, graphite. The unit cell of graphene comprises of two carbon atoms which form an extended honeycomb lattice (Fig. 3A). Graphene is also one of the strongest known materials with an elastic modulus of $1000 \mathrm{GPa}$. Single-layer graphene can be readily synthesized in large sheets (several $\mathrm{m}^{2}$ in area) on a roll-to-roll basis by the chemical vapor deposition (CVD) process. ${ }^{[15]}$ The fact that the graphene sheet is only one atom thick and is chemically, thermally and mechanically robust, makes graphene extremely attractive for the membrane application. However, the electron density gap in graphene's honeycomb lattice is too small to allow molecular translocation. Therefore, one needs to etch the graphene lattice to form molecular-sized nanopores (Fig. 3B). Successful incorporation of molecularsized pores in graphene even at a small porosity leads to a large molecular permeance attributing to the ultrashort diffusion path of the permeating molecule. This has been clearly demonstrated by a number of molecular-dynamics simulations. For example, Jiang and co-workers calculated $\mathrm{CO}_{2}$ permeance of $2.9 \times 10^{5} \mathrm{GPU}$, and a $\mathrm{CO}_{2} / \mathrm{N}_{2}$ selectivity of 300 from a nanoporous graphene lattice hosting a pore-density of $0.04 \mathrm{~nm}^{-2}$ (equivalent to a porosity of 0.01). ${ }^{[16]}$ In their study, each pore was made by missing ten carbon atoms. $\mathrm{A} \mathrm{CO}_{2} /$ $\mathrm{N}_{2}$ selectivity greater than 1000 has been experimentally observed from bilayer graphene microballons indicating that such high-performance membranes may indeed be achievable. ${ }^{[17]}$ In comparison, the stateof-the-art polymeric membranes yield a much lower $\mathrm{CO}_{2}$ permeance $(100-1000$ GPU with the selective layer thickness of $0.1-1.0 \mu \mathrm{m})$. The required membrane area for a separation process follows an inverse relationship with the membrane permeance, and therefore the ultrahigh permeance of the nanoporous graphene membrane is expected to drastically reduce the overall membrane area. For example, for a separation process where $1000 \mathrm{~m}^{2}$ of a polymeric membrane is required, only 1-10 $\mathrm{m}^{2}$ of nanoporous graphene membrane could be sufficient. As a result, even though the graphene membrane may turn out to be much more expensive on a specific area basis, the overall cost of the membrane is expected to be at parity with that of the polymeric membranes. For example,
Fig. 1. Gas and vapor transport across nanoporous 2D membranes. A) A cartoon of the nanoporous 2D membrane. B) The concentration profile of a permeating molecule across a thin film. C) The mechanism of molecular transport across the nanoporous 2D membranes. 
consider the case of $\mathrm{CO}_{2}$ capture from flue gas (post-combustion carbon capture) in a power plant. It has been shown that a membrane with a minimum $\mathrm{CO}_{2}$ permeance of $2000 \mathrm{GPU}$ and minimum $\mathrm{CO}_{2} / \mathrm{N}_{2}$ mixture separation factor of 30 can be more energy-efficient than the conventional aminebased $\mathrm{CO}_{2}$ scrubbing. ${ }^{[4]}$ Theoretical and experimental evidence point out that graphene can indeed be engineered to obtain $\mathrm{CO}_{2}$ permeance of $100,000 \mathrm{GPU}$ and $\mathrm{CO}_{2} /$ $\mathrm{N}_{2}$ mixture separation factor of 30 . As a result, only a small membrane area $\left(200 \mathrm{~m}^{2}\right)$ will be needed, while the overall capital cost (CAPEX) would be parity to that of the polymeric membranes (Table 1). This also has a huge implication in the scale-up of the nanoporous 2D membranes, where a small membrane area (of the order of 10-100 $\mathrm{m}^{2}$ ) will be sufficient to handle the separation needs of a medium to largescale chemical plant. As an added bonus, the graphene membranes are expected to outperform their polymeric counterparts in terms of durability especially at high pressure and temperature conditions.

Graphene was isolated from graphite for the first time in 2004 by Geim and Novoselov leading to the Nobel prize in Physics in 2010. The chemical synthesis and transfer of a large single-layer graphene film were reported for the first time in 2009. ${ }^{[18]}$ Since then, there has been a flurry of efforts to develop high-quality singlelayer graphene membranes. ${ }^{[19]}$ However, to date, a high-quality (free of cracks and tears), large-area, single-layer graphene membrane has remained elusive. One of the fundamental challenges in achieving a high-performance graphene membrane is the incorporation of a high-density of molecular-sized pores. ${ }^{[19]}$ For the separation of gases and vapors, this corresponds to etching pores with a mean pore-size of 0.3 to $1.0 \mathrm{~nm}$, while maintaining a narrow pore-size-distribution (PSD). Typically, even a small percentage $(0.1 \%)$ of non-selective pores reduces the separation selectivity because of the onset of viscous and Knudsen flow through these pores (Eqn. (1) and (2), respectively). There are several chemical and physical routes to etch the graphene lattice including exposure to UV/ ozone, oxidative plasma, the bombardment of ions and energetic electrons, electrical pulse, etc. However, all of these methods lead to a tradeoff between the pore-density and the PSD (Fig. 3C, i). Usually, at a relatively short etching time, one can etch a low-density of pores with a narrow PSD. However, subsequent etching to increase the pore-density also leads to expansion of the existing pores. This is because of the high reactivity of $\mathrm{sp}^{3}$-hybridized carbon atoms at the pore-edge. According to the activation energies of nucleation and pore-propagation in graphite, ${ }^{[20]}$ the etch-

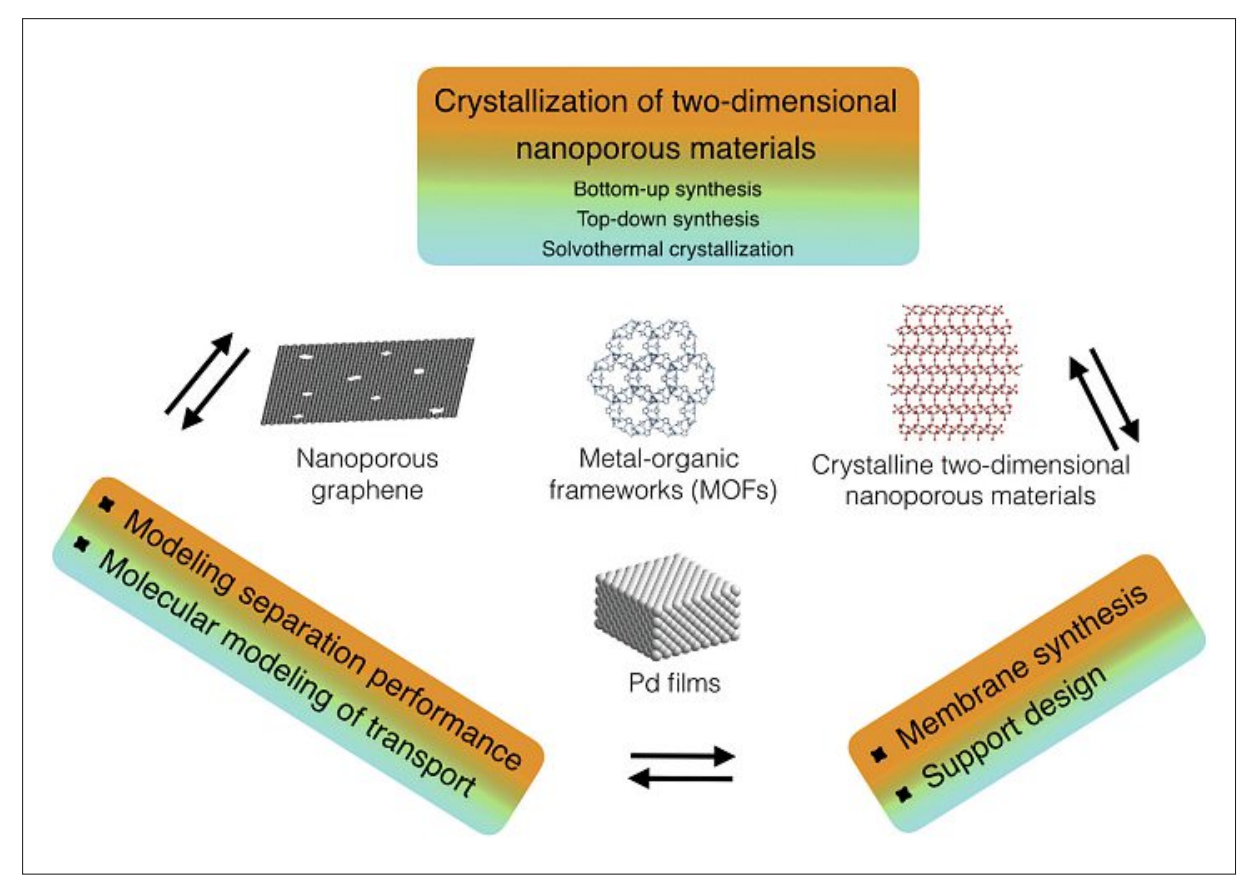

Fig. 2. Summary of research activities at the Laboratory of Advanced Separations (LAS).
A

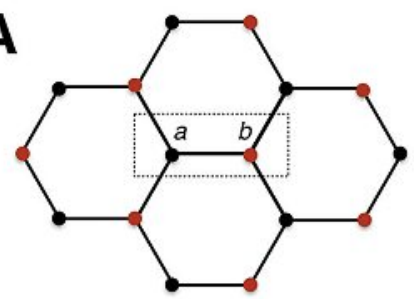

C

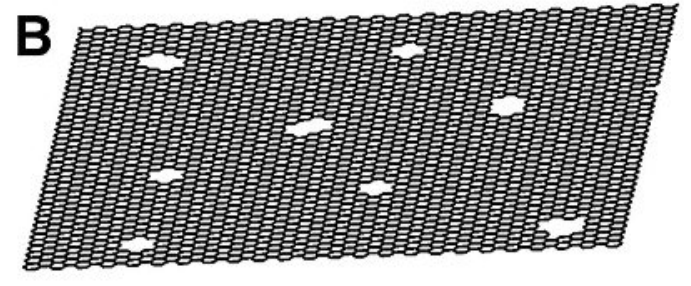

(ii) Chemistry for tunable but narrow pore-size distribution - Incorporation of reactive sites - Selective etching of reactive sites

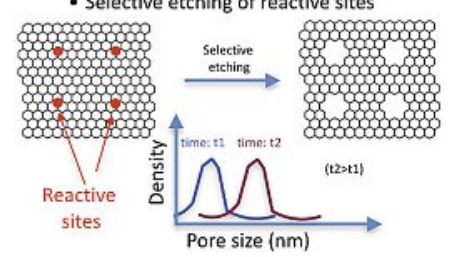

Fig. 3. Application of graphene in nanoporous $2 \mathrm{D}$ membranes. A) The crystal structure of graphene with each unit cell comprising of two atoms ( $a$ and $b)$. B) A schematic of nanoporous graphene. C) A comparison of the conventional approach (i) and the lead approach at LAS to generate tunable pores with a narrow PSD in graphene.

Table 1. Scale-up scenario for the capture of 0.5 million tonnes/year of $\mathrm{CO}_{2}$ (typical emission from a natural gas power plant).

\begin{tabular}{|l|c|c|c|c|}
\hline & $\begin{array}{c}\mathbf{C O}_{2} \\
\text { Permeance } \\
{[\mathbf{G P U}]}\end{array}$ & $\begin{array}{c}\text { Area needed } \\
{\left[\mathbf{m}^{2}\right]}\end{array}$ & $\begin{array}{c}\text { Cost } \\
{\left[\mathbf{C H F} / \mathbf{m}^{2}\right]}\end{array}$ & $\begin{array}{c}\text { CAPEX } \\
{[\mathbf{C H F}]}\end{array}$ \\
\hline Polymeric & 1000 & 20000 & $50-500$ & $1-10$ million \\
\hline $\begin{array}{l}\text { Nanoporous } \\
\text { Graphene }\end{array}$ & 100000 & 200 & $1000-10000$ & $0.2-2$ million \\
\hline
\end{tabular}


ing rate of the edge-atoms can be several orders of magnitude higher than that of the basal-plane-atoms. To solve this problem, LAS is developing novel etching chemistries that target the basal plane in a controlled manner. The leading approach is to engineer the reactivity of the graphene lattice by functionalization (Fig. 3C, ii). For this, LAS is studying the reactivity of functionalized graphene (basal plane and pore-edge) and is developing kinetic models based on the etching rate and the activation energy. In addition, LAS is employing a novel approach involving competitive growth and etching of graphene pores in the CVD reactor. The largest benefit of the approach is that one can generate a highdensity of large nanopores in graphene by the conventional etching processes, and subsequently, narrow down the PSD by recrystallizing the graphene in a controlled growth/etching environment.

Recently, we reported the structure and the origin of the intrinsic defects in CVD graphene. ${ }^{[21]}$ A scanning tunneling microscopy (STM) study indicated that the intrinsic defects of the CVD graphene are comprised of missing 10-16 carbon atoms (Fig. 4A). Similarly-sized nanopores have shown promising performance in gas separation (molecular simulations). ${ }^{[22-25]}$ The origin of intrinsic defects is of fundamental importance to understand the CVD process, and is expected to facilitate the tuning of PSD and pore-density. It is highly probable that these defects are incorporated in the graphene lattice by etching inside the CVD furnace. Here, the key challenge is to identify the type of the etchant and the nature of the etching chemistry. Several reports point to the fact that hydrogen, usually added to the CVD environmental, can etch graphene.[26,27] However, to etch graphene, the molecular hydrogen must first dissociate into the atomic hydrogen on the surface of graphene. This is highly unlikely at the CVD conditions. Hydrogen does play an important role in the crystallization of graphene by controlling the relative population of the carbon radicals obtained from the dehydrogenation of hydrocarbon precursors (typically $\mathrm{CH}_{4}$ ). Since hydrogen is also the product of the dehydrogenation reaction, a high hydrogen concentration in the CVD reactor would promote a relatively higher population of partially hydrogenated radicals (for example, $\mathrm{CH}^{*}$, $\mathrm{CH} 2 *, \mathrm{CH}_{3}$ ) compared to the completely dehydrogenated $\mathrm{C}^{*}$ radical. Therefore, changing hydrogen concentration in the reactor can potentially alter the crystallization pathway and the final morphology of the graphene grains.

A systematic study on the etching of graphene in the presence of hydrogen and residual oxygen in the CVD reactor revealed that it is residual oxygen and not hydrogen that is responsible for the etching of graphene.[21] Soaking graphene in an oxygen-depleted hydrogen atmosphere $\left(\mathrm{H}_{2} / \mathrm{O}_{2}\right.$ ratio of 7600 , pressure of 108 torr $)$ at $1077{ }^{\circ} \mathrm{C}$ for up to $3 \mathrm{~h}$ neither etched graphene edges nor increased the defect density (Fig. 4B). However, soaking graphene in a higher oxygen concentration $\left(\mathrm{H}_{2} / \mathrm{O}_{2}\right.$ ratio of 300) for just $1 \mathrm{~h}$ etched graphene completely (Fig. 4B). Increasing the oxygen concentration in the reactor $\left(\mathrm{H}_{2} / \mathrm{O}_{2}\right.$ ratio of 10) for just 1 min increased the defect-density in CVD graphene by 7-fold

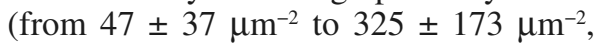
Fig. 4C).

Apart from the challenge of incorporating molecular-sized pores in graphene, another challenge in the development of suspended single-layer graphene films is to prevent cracks and tear during the transfer of CVD graphene to a porous support. The CVD process is by far the most robust technique to synthesize single-layer graphene in a scalable manner. The roll-toroll synthesis of the single-layer graphene film by the CVD process has been recently demonstrated. $[15,28,29]$ The graphene growth rates can be quite fast (typically $1-10 \mu \mathrm{m} /$ min) on a $\mathrm{Cu}$ foil, and even faster on $\mathrm{Cu}$ foils alloyed with $\mathrm{Ni}$ (larger than $100 \mu \mathrm{m} /$ min). Due to this and a high nucleation density typically observed in the low-pressure CVD (typically several nuclei/ $\mu \mathrm{m}^{2}$ ), a polycrystalline graphene film can cover the entire surface of the $\mathrm{Cu}$ foil in less than $1 \mathrm{~min}$. To fabricate membranes, the CVDderived graphene film needs to be transferred from the metal foil to a porous support. This must be carried out without generating cracks or tears in the graphene film, otherwise, the large defects dominate the molecular transport leading to poor separation selectivity. Recently, the Agrawal group successfully transferred large (1-2 $\mathrm{mm}$ in size) graphene grains onto a porous $\mathrm{W}$ support comprising of a single $5-\mu \mathrm{m}$ hole.[21] The millimeter-sized grains and a careful transfer ensured that the $\mathrm{W}$ pore was covered entirely with a single graphene grain, allowing a study of gas transport and stability of a CVD-derived grain-boundary-free graphene. Gas flux was readily detected from the bare $\mathrm{W}$ support and when cracks developed during graphene transfer. However, no gas flux was observed when the transferred grain was crack-free, and gas transport from the intrinsic defects could not be inferred. This was because of a low density of intrinsic defects $(47 \pm 37$ $\left.\mu \mathrm{m}^{-2}\right)$, a small membrane area ( $5 \mu \mathrm{m}$ in diameter), and the restricted detection limit of the mass spectrometer. This implies that single-layer graphene with a low-density of intrinsic-defects can act as an excellent gas barrier. The suspended graphene film remained intact even after exposing it to an extremely oxidative environment (15 mole\% ozone at $200{ }^{\circ} \mathrm{C}$ for $3 \mathrm{~min}$ ), and repeated temperature cycling from 25 to $200{ }^{\circ} \mathrm{C}$ for several weeks, indicating remarkable chemical and mechanical stability of graphene. The promising stability of graphene is ideal for its use in membrane applications. Currently, LAS is developing methods to increase the defect-density in graphene and transfer large-area graphene on a porous support to study molecular transport from graphene nanopores. LAS is developing a novel transfer-technique improving the stress-bearing capacity of

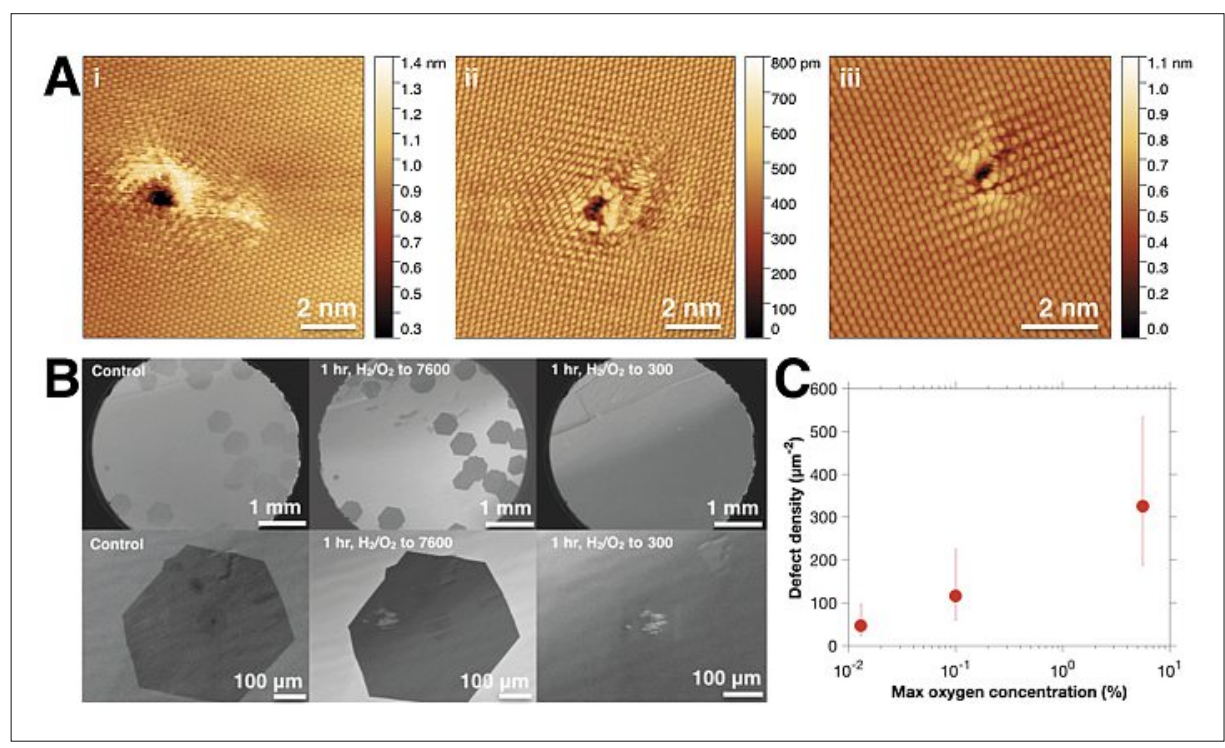

Fig. 4. Structure and origin of intrinsic defects in CVD graphene. A) STM images of the assynthesized CVD graphene revealing intrinsic defects made of missing 10-16 carbon atoms. B) Exposure of graphene crystals in various $\mathrm{H}_{2} / \mathrm{O}_{2}$ environments revealed the predominant role of $\mathrm{O}_{2}$ in graphene etching. C) Increase in the defect-density of graphene as a function of the $\mathrm{O}_{2}$ concentration in the CVD reactor. Obtained with permission. Reprinted with permission from ref. [21]. Copyright (2017) American Chemical Society. 
the suspended graphene film. The lead approach is to coat an ultrathin mechanically reinforcing, porous film on top of the graphene film.

\subsection{Ultrathin Polycrystalline MOF Films}

MOFs are porous, crystalline coordination polymers comprising of metal nodes coordinated by organic ligands. The reticular chemistry employed in the synthesis of MOF has afforded thousands of MOF structures of which several are chemically and thermally stable, and possess molecular-size apertures. ${ }^{[30]}$ The synthesis of MOFs can be extremely rapid. For example, the induction time for the crystallization of ZIF-8 is only a few seconds at room temperature. This makes application of MOFs in membranes highly attractive. One of the challenges in achieving size-selective MOF membranes is that MOFs undergo structure dilation upon exposure to the guest molecules, and as a result, a sharp molecular cutoff is not obtained. Several MOFs can accommodate molecules much larger than what one estimates based on the crystallographically determined lattice parameters. Interestingly, this has also been an opportunity area for the kinetic separation of gases. For example, the crystallographically determined pore-aperture of ZIF-8 is $0.34 \mathrm{~nm}$. However, both propane and propylene (kinetic diameters of 0.44 and 0.42 $\mathrm{nm}$, respectively) can diffuse through the ZIF-8 lattice, albeit with different diffusivity. Here, one can exploit the diffusivity difference between the molecules to separate propylene from propane. For instance, the ratio of diffusivity of propylene and propane in the ZIF-8 lattice is 125 at $30^{\circ} \mathrm{C}$. [31] Exploiting structure dilation, recently, an ultramicroporous MOF (ZIF-7, phase III) possessing a pore aperture of $0.21 \mathrm{~nm}$ was successfully employed to separate $\mathrm{H}_{2}$ from $\mathrm{CO}_{2}$ (kinetic diameters of $\mathrm{H}_{2}$ and $\mathrm{CO}_{2}$ are 0.29 and $0.33 \mathrm{~nm}$, respectively) with $\mathrm{H}_{2} /$ $\mathrm{CO}_{2}$ selectivity up to 300 , and $\mathrm{a}_{2}$ permeance up to 3000 GPU. [32]

Single-phase, polycrystalline $\mathrm{MOF}$ films can be highly attractive for molecular separation. The presence of nanoporous channels/apertures in MOF films results in orders of magnitude higher permeance and higher selectivity than that obtained from the sorption-diffusion of molecules in the free volume of the polymeric films. However, the synthesis of polycrystalline MOF films is much more challenging than that of the polymer films. Recently, a hybrid approach, mixed-matrix-membranes (MMM) has been successfully used to develop high-quality membranes. Here, MOF crystals are dispersed in a suitable polymer matrix, improving the separation selectivity of the polymeric membrane. However, the MMM approach has a lower processability than that of a pure-phase polymer film. The skin-layer thickness of the MMM is restricted by the MOF crystals. As a result, it is almost impossible to synthesize a 100-nm-thick selective film in the case of the MMM approach, whereas one can synthesize polymeric membranes with 100-nm-thick skin layers on a routine basis. As a result, although MMM offers higher permeability, the actual permeance of the membrane tends to be lower.

LAS is focused on developing methods to reduce the complexity in the synthesis of polycrystalline MOF films. Here, the key challenges are i) synthesis of ultrathin films to obtain a high permeance, b) reduction of pinhole and grain-boundary defects to improve the separation selectivity, c) improvement in the stability of the films, including that of the grain boundaries. In this pursuit, LAS has developed a novel crystallization approach, referred to as electrophoretic nuclei assembly for crystallization of highly-intergrown thinfilms (ENACT). ${ }^{[33]}$ Here, a high density of heterogeneous nucleation on a substrate is achieved directly from the MOF precursor sol by attracting the nuclei towards a substrate under the influence of an electric field (Fig. 5A). An advantage of this approach is that one can avoid the lengthy preparation of the seed crystals and the seed film typically used for the popular seeded secondary growth for the crystallization of high-performance MOF membranes. The seeded secondary growth approach involves crystallization of seed crystals in high yield (usually a long wait time), separation of seed crystals from the growth solution, dispersion and stabilization of seed crystals in an appropriate solvent, and finally coating a thin layer of seed on the substrate. The ENACT approach skips these steps. An ultrathin ZIF-8 film could be synthesized directly from a dilute precursor sol by simply immersing the substrate in the precursor sol followed by application of an electric field for a few minutes. This leads to the deposition of a highly-dense nuclei film on the substrate. Ultrathin, pinhole-free, and highly intergrown ZIF-8 films were obtained by simply leaving the nuclei film in the precursor sol for up to $10 \mathrm{~h}$. Another advantage of the ENACT approach lies in its versatility. Support modification, typically employed for the synthesis of MOF membranes, was not required, and as-obtained commercial supports (anodic aluminum oxide and porous polyacrylonitrile film) could be directly used. The approach can be used to prepare films from several MOF frameworks. For instance, both ZIF-8 and ZIF-7 film could be prepared. A variety of sub- strates could be employed including ceramic (anodic aluminum oxide), polymer (polyacrylonitrile), nanoporous carbon, graphene and metal ( $\mathrm{Cu}$ foil). The process could be carried out on both porous and non-porous substrates allowing us to form films for a range of applications including membranes, sensors, etc. Since electrophoretic deposition allowed for a high heterogeneous nucleation density, pinhole defects in membranes reduced substantially even when the membrane was only 500-nm-thick (Figs. 5B and C). This led to high-quality ZIF-8 membranes which yielded one of the best separation performance (Figs. 5D-F) in the separation of $\mathrm{H}_{2} / \mathrm{C}_{3} \mathrm{H}_{8}\left(\mathrm{H}_{2}\right.$ permeance of $8 \times 10^{-6}$ mole $\mathrm{m}^{-2} \mathrm{~s}^{-1} \mathrm{~Pa}^{-1}$ and selectivity up to 2655) and $\mathrm{C}_{3} \mathrm{H}_{6} / \mathrm{C}_{3} \mathrm{H}_{8}\left(\mathrm{C}_{3} \mathrm{H}_{6}\right.$ permeance of $10^{-7}$ mole $\mathrm{m}^{-2} \mathrm{~s}^{-1} \mathrm{~Pa}^{-1}$ and selectivity of 31.6).

Ideally, one would like to synthesize ZIF-8 membranes with $\mathrm{C}_{3} \mathrm{H}_{6} / \mathrm{C}_{3} \mathrm{H}_{8}$ selectivity $>100$. This can only happen if the defects in the polycrystalline MOF film can be reduced substantially. For this, LAS is investigating methods to control the Ostwald ripening which was observed during the growth stage of the nuclei film. In general, crystallization approaches like ENACT offer a novel solution to tune the crystallization process allowing the fabrication of ultrathin polycrystalline films. LAS is now reapplying the ENACT approach to synthesize a range of MOF structures in a thin polycrystalline film.

\subsection{Exfoliated Nanoporous 2D Nanosheets: Zeolite Nanosheets and their Membranes}

Zeolites are porous, crystalline metallosilicates comprised of a periodic arrangement of corner-shared silicate tetrahedra. The metal sites (M) are usually Al, $\mathrm{Ti}, \mathrm{Ge}, \mathrm{Sn}$, etc., and are primarily incorporated in the zeolitic framework for application in catalysis and adsorption, ${ }^{[34]}$ or for a post-synthetic structural modification. ${ }^{[35]}$ For application in membranes, siliceous zeolites ( $\mathrm{Si} / \mathrm{M}=$ infinity) are more suitable (barring application in water-selective pervaporation), due to the higher stability of the siliceous zeolites. The zeolitic framework is made up of covalent bonds (-O-Si-O-), which makes their lattice relatively rigid, and as a result, a sharp sizeselectivity can be expected from a zeolite membrane. The covalent nature of the bonding also imparts exceptional chemical and thermal stability to the zeolites, making zeolite membranes highly attractive for separation of molecules in harsh chemical and/or thermal conditions. Currently, there are 228 zeolite frameworks that are officially recognized on the webpage of International Zeolite Association. ${ }^{[36]}$ Out of these, several frameworks (MFI, LTA, SOD, CHA, DDR, FAU, MOR, BEA, 


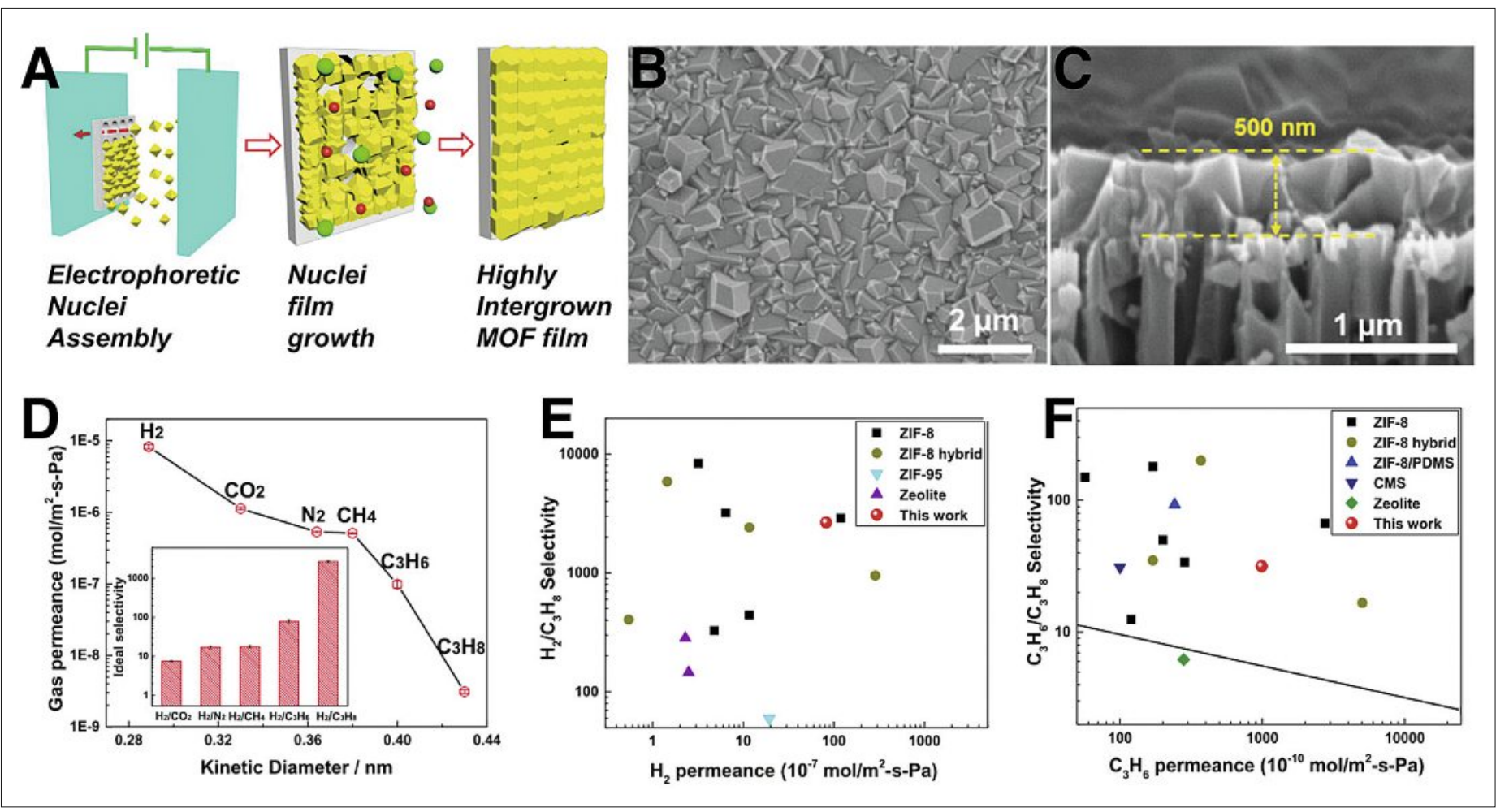

Fig. 5. Novel ENACT technique for the synthesis of highly-intergrown ultrathin MOF films. ${ }^{[33]}$ A) Schematic showing the implementation of ENACT. A top-view and a cross-sectional image of the ZIF-8 membrane is shown in (B) and (C), respectively. D) The permeance of several gases as a function of kinetic diameter, demonstrating the kinetic separation of gases. The inset reveals the ideal selectivity for several gas pairs. E,F) The performance of the ZIF-8 membrane prepared by ENACT process is compared with that of other membranes in the literature. Figure reproduced from ref. [33]. Copyright Wiley-VCH Verlag GmbH \& Co. KGaA. Reproduced with permission.

MWW, etc.) have been synthesized in polycrystalline film morphology.

The first zeolite membranes were developed more than two decades ago, and yet they have not been commercialized to an extent that one expects based on their high performance. One of the biggest challenges in the scale-up of zeolite membranes is the lack of reproducibility in the synthesis of polycrystalline zeolite films. This is because of a complicated crystallization mechanism which involves a complex aggregated growth mechanism. ${ }^{[37]}$ The need to crystallize defect-free, thin zeolite film on a porous support adds to the complexity because one needs to eliminate pinhole and reduce grain-boundary defects. Such defects may arise because of a preferential growth direction, poor heterogeneous nucleation, seed dissolution, Ostwald ripening, etc. The complexity can be completely eliminated if a defect-free zeolite film can be fabricated by simply coating a dispersion of pre-synthesized zeolite crystals. However, packing colloidal crystals perfectly is a daunting task because this involves completely eliminating the intercrystal gaps. A high packing fraction can be obtained if the colloidal crystals are of cuboidal shape (like an assembly of bricks). A defect-free film can be achieved if the constituent crystals are flexible such that any defect in the packing, formed during the crystal assembly, can be ef- fectively sealed. Keeping this in mind, 2D zeolite crystals (a few unit cells thick and micron-sized in the lateral dimension) are an ideal building block, and therefore the synthesis of high-aspect-ratio (hence flexible), 2D zeolite crystals can pave the way for the synthesis of reproducible, highperformance zeolite membranes.[38] Since the zeolite surface terminates with silanol groups, one could condense these groups between two overlapping nanosheets to form a covalent bond between the crystals, improving the sealing and mechanical properties of the coating.

Pursuing the concept of $2 \mathrm{D}$ zeolite nanosheets, we developed MFI- and MWWnanosheets with nanosheets being 1.5 and 1.0 unit cells thick, respectively. ${ }^{[39]} \mathrm{MWW}$ consists of two sets of independent pore channels accessible through 10-memberring windows (made of 10 corner-shared tetrahedra). MWW is typically synthesized by the topotactical condensation of unitcell-thick layers along the $c$-axis present in its precursor (MCM-22 (P) or ITQ-1 precursor). ${ }^{[40]}$ The constituent layer by itself is microporous, comprising of dumbbellshaped pores (12-member-ring pore opening with 6-member-ring constriction at the center). Here, the 6-member-ring (MR) window with an effective electron-density-gap close to $0.3 \mathrm{~nm}$ is attractive for the molecular-sieving of $\mathrm{He}, \mathrm{Ne}$ and $\mathrm{H}_{2}$ from larger molecules $\left(\mathrm{CO}_{2}, \mathrm{~N}_{2}, \mathrm{CH}_{4}\right.$, $\mathrm{Ar}$, etc. $)$.
MFI comprises of two separate set of pore channels accessible through a 10-memberring window. One of the channels runs straight along the $b$-axis, while the other channel runs zig-zag along the $a$-axis. The electron-density gap of the channel window is ca. $0.55-0.60 \mathrm{~nm}$, making them ideal for the separation of benzene derivatives (for example, $p$-xylene from $o$-xylene and $m$-xylene). However, unlike MWW, MFI is crystallized in a three-dimensional morphology, which makes the synthesis of the 2D MFI-nanosheets highly improbable. In 2009, Ryoo and co-workers reported a surfactant-templating approach to synthesize multilamellar MFI where each layer was 1.5 unit-cell thick along the $b$-axis. ${ }^{[41]}$ This provided an opportunity to synthesize the 2D MFI-nanosheets by exfoliation.

The exfoliation of zeolitic layers can prove to be challenging because of a much higher binding energy between the layers compared to that between the graphitic layers. The latter can be readily exfoliated in a solvent by matching the solubility parameters. ${ }^{[42]}$ The high binding energy of the zeolitic layers arises from the contributions from the van der Waal's as well as the electrostatic interaction between the layers. As a result, the zeolitic layers do not exfoliate in solvents. To assemble thin defect-free films from exfoliated zeolite nanosheets, one has to develop a mild exfoliation process, that on one hand is 
scalable and provides a good yield, and on another hand, conserves the crystalline structure and the sheet-like morphology of the nanosheets. ${ }^{[39]}$ For example, Corma and co-workers reported exfoliation of the MWW layers by swelling them using surfactant at high $\mathrm{pH}$ and temperature followed by sonication at high $\mathrm{pH}$ and temperature. ${ }^{[43]}$ Here, the harsh conditions led to a considerable silica dissolution, and the exfoliated material lost their rigid sheet-like morphology. Therefore, to achieve exfoliation, a mild process was developed. First, the interlayer spacing of as-synthesized zeolite was increased by surfactant swelling at room temperature. As a result of swelling with cetyltrimethyl ammonium bromide (CTAB), the interlayer spacing of the MWW precursor was increased by $1.8 \mathrm{~nm}$. The as-synthesized multilamellar MFI layers already comprised of a large interlayer spacing, 3.0 $\mathrm{nm}$, due to the use of long-tailed structure directing agent (SDA), and therefore there was no need for the surfactant-swelling step. Exfoliation of the swollen layers was carried out by shearing them in a polymer melt. ${ }^{[39]}$ Here, the polymer chains intercalate in the gallery spacing and subsequently exfoliate the layers when a high shear stress is applied by bringing the melt temperature close to the glass tran- sition temperature. A solution-processing route was developed to purify the exfoliated nanosheets, removing polymer and unexfoliated nanosheets. The residual surfactant and SDA were removed by acid treatment, yielding a dispersed suspension of zeolite nanosheets in ethanol. [44] The resulting nanosheets were highly crystalline, maintained the microporous structure and the sheet-like morphology (Figs. 6A-D). Ultrathin zeolite films (monolayer-thick to 50 -nm-thick) were fabricated by stacking the nanosheets on a porous support by filtration or by the Langmuir-Schaffer technique. To reduce the intersheet gaps, solvothermal and gel-free secondary growth techniques were developed which promoted in-plane crystal growth (Figs. $6 \mathrm{E}$ and F). ${ }^{[45]}$ High-quality membranes could be prepared by this process which led to record-high gas and vapor permeances with an extremely high separation selectivity ( $p$-xylene/o-xylene selectivity reaching 1000, Fig. 6G).

In general, the overall process of preparing a dispersed suspension of exfoliated nanosheets using swelling, melt-compounding and solution processing is highly versatile, and LAS is extending this for the exfoliation of layered silicates (RUB15), ${ }^{[47]}$ and $\mathrm{g}-\mathrm{C}_{3} \mathrm{~N}_{4}$ (poly(triazine imide) and $\left.\mathrm{C}_{2} \mathrm{~N}\right) \cdot{ }^{[48,49]}$

\section{Future Perspectives}

Membrane-based separations have a bright future owing to their high energyefficiency, clean operation, straightforward operation in a decentralized fashion, and their potential for process intensification. Nanoporous 2D films, if synthesized with a high porosity in a defect-free morphology, are destined to be the ultimate membranes, thanks to their promise of ultrahigh permeance and attractive separation selectivity. A successful synthesis of such membranes offers a great opportunity for an interdisciplinary research in chemistry, material science, and chemical engineering. There are a wide-range of separation challenges in the chemical industry, requiring the development of membranes with precise pore-size ranging from 0.25 to $1.0 \mathrm{~nm}$. Here, nanoporous graphene membranes will be especially attractive because of the tunability of their PSD. However, methods to incorporate precise nanopores at a high pore-density in graphene are still lacking. The emerging classes of crystalline, layered, microporous materials (MOFs, covalent organic frameworks (COFs), porous aromatic frameworks (PAFs), g- $\mathrm{C}_{3} \mathrm{~N}_{4}$, etc.) capable of carrying out molecular-sieving are also interesting in this regard. However, a mild
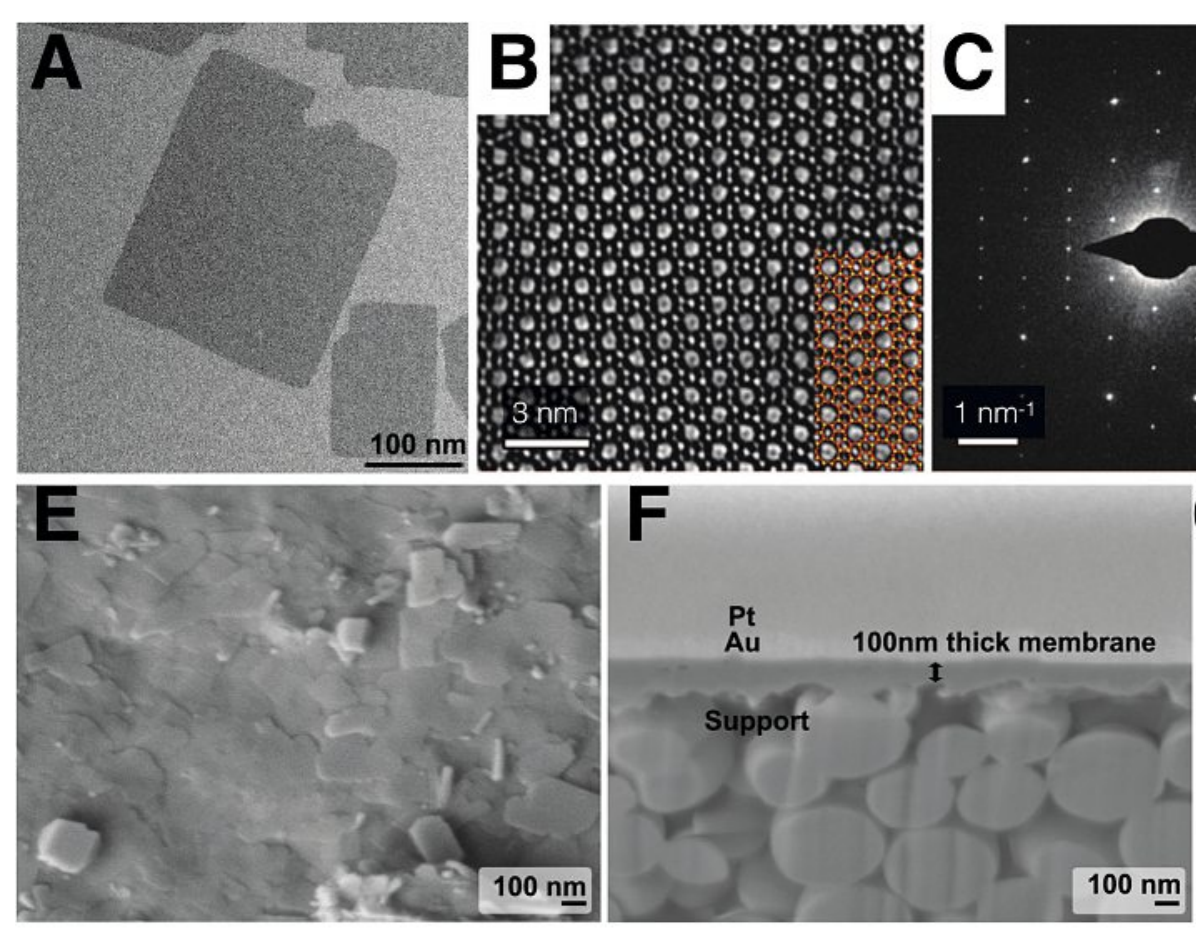
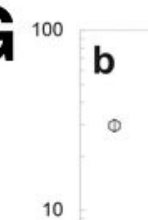

क

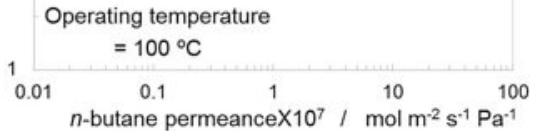

Fig. 6. Exfoliated zeolite nanosheets and resulting ultrathin membranes. Transmission electron microscopy (TEM) ${ }^{[45]}$ (A) and Bragg-filtered highresolution TEM (HRTEM) ${ }^{[4]}$ images of 1.5-unit-cell-thick MFI-nanosheets (B). The crystal structure of MFI nanosheet along the [010] zone axis is overlaid on top of the HRTEM image in (B). C) Electron diffraction (ED) pattern of MFI nanosheets along the [010] zone axis. ${ }^{[46]}$ Both HRTEM and ED indicate the high crystallinity of MFI-nanosheets. D) Crystal structure of MFI (i) and MWW (ii) nanosheets. $\left.{ }^{[39]} \mathrm{E}-\mathrm{F}\right)$ 100-nm-thick membrane prepared by gel-less secondary growth of MFI nanosheet film. ${ }^{[45]} \mathrm{G}$ ) Comparison of the $n$-butane/i-butane separation performance for several MFI membranes reported in the literature. ${ }^{[45]}$ The separation factor (SF) is plotted against $n$-butane permeance. Nanosheet-derived membranes lead to the best performance (two nanosheet-derived membranes indicated by the empty and the filled star markers). (A), (E), (F), and (G) reproduced from ref. [45], with permission from Wiley-VCH. (D) reproduced from ref. [39]. Reprinted with permission from AAAS. 
technique to exfoliate their layered precursor to the single-layer, and the stability of the exfoliated layer remains unknown. The polycrystalline MOF membranes will greatly benefit from a scalable crystallization route that promotes in-plane growth and reduces the grain-boundary defects. Finally, the holy grail of the nanoporous 2D film will be the development of bottom-up techniques (such as CVD) capable of depositing a nm-thick nanoporous film possessing an ordered porous structure with a sub-nanometer pore-size.

\section{Acknowledgements}

We acknowledge the generous support from our host institution, EPFL, as well as the ETH Board. We also acknowledge funding from the Swiss National Science Foundation (Assistant Professor Energy Grant, project number: PYAPP2_173645/1), our industrial partner GAZNAT, and the Swiss Competence Center for Energy Research - Efficiency in Industrial Processes (SCCER-EIP, Phase II). We also acknowledge support from the "EPFL Fellows" fellowship program co-funded by Marie Sklodowska-Curie, which received funding from the European Union's Horizon 2020 Framework Programme for Research and Innovation under grant agreement 665667.

Received: February 23, 2018

[1] J. L. Humphrey, G. E. Keller, 'Separation Process Technology', McGraw-Hill, 1997.

[2] D. S. Sholl, R. P. Lively, Nature 2016, 532, 435

[3] T. M. McDonald, J. A. Mason, X. Kong, E. D. Bloch, D. Gygi, A. Dani, V. Crocellà, F. Giordanino, S. O. Odoh, W. S. Drisdell, B. Vlaisavljevich, A. L. Dzubak, R. Poloni, S. K. Schnell, N. Planas, K. Lee, T. Pascal, L. F. Wan, D. Prendergast, J. B. Neaton, B. Smit J. B. Kortright, L. Gagliardi, S. Bordiga, J. A. Reimer, J. R. Long, Nature 2015, 519, 303.

[4] S. Roussanaly, R. Anantharaman, K. Lindqvist, H. Zhai, E. Rubin, J. Memb. Sci. 2016, 511, 250

[5] L. M. Robeson, J. Memb. Sci. 2008, 320, 390.

[6] D.-Y. Koh, B. A. McCool, H. W. Deckman, R. P. Lively, Science 2016, 353, 804.

[7] M. Y. Jeon, D. Kim, P. Kumar, P. S. Lee, N. Rangnekar, P. Bai, M. Shete, B. Elyassi, H S. Lee, K. Narasimharao, S. N. Basahel, S. Al-Thabaiti, W. Xu, H. J. Cho, E. O. Fetisov, R. Thyagarajan, R. F. Dejaco, W. Fan, K. A. Mkhoyan, J. I. Siepmann, M. Tsapatsis, Nature 2017, 543, 690 .

[8] E. Favre, J. Memb. Sci. 2007, 294, 50.
[9] F. V Lima, P. Daoutidis, M. Tsapatsis, J. J. Marano, Ind. Eng. Chem. Res. 2012, 51, 5480

[10] H. B. Park, J. Kamcev, L. M. Robeson, M. Elimelech, B. D. Freeman, Science 2017, 356, eaab0530.

[11] H. B. Park, C. H. Jung, Y. M. Lee, A. J. Hill, S. J. Pas, S. T. Mudie, E. Van Wagner, B. D. Freeman, D. J. Cookson, Science 2007, 318, 254.

[12] P. M. Budd, E. S. Elabas, B. S. Ghanem, S Makhseed, N. B. McKeown, K. J. Msayib, C. E. Tattershall, D. Wang, Adv. Mater. 2004, 16, 456.

[13] K. K. Tio, S. S. Sadhal, Appl. Sci. Res. 1994, 52, 1.

[14] K. Celebi, J. Buchheim, R. M. Wyss, A. Droudian, P. Gasser, I. Shorubalko, J.-I. Kye, C. Lee, H. G. Park, Science 2014, 344, 289.

[15] T. Kobayashi, M. Bando, N. Kimura, K. Shimizu, K. Kadono, N. Umezu, K. Miyahara, S. Hayazaki, S. Nagai, Y. Mizuguchi, Y. Murakami, D. Hobara, Appl. Phys. Lett. 2013, 102, 23112.

[16] H. Liu, S. Dai, D. Jiang, Nanoscale 2013, 5, 9984.

[17] S. P. Koenig, L. Wang, J. Pellegrino, J. S. Bunch, Nat. Nanotechnol. 2012, 7, 728.

[18] X. Li, W. Cai, J. An, S. Kim, J. Nah, D. Yang, R. Piner, A. Velamakanni, I. Jung, E. Tutuc, S. K. Banerjee, L. Colombo, R. S. Ruoff, Science 2009, 324, 1312.

[19] L. Wang, M. S. H. Boutilier, P. R. Kidambi, D. Jang, N. G. Hadjiconstantinou, R. Karnik, Nat. Nanotechnol. 2017, 12, 509.

[20] X. Chu, L. D. Schmidt, Surf. Sci. 1992, 268, 325.

[21] K. V. Agrawal, J. D. Benck, Z. Yuan, R. P. Misra, A. Govind Rajan, Y. Eatmon, S. Kale, X. S. Chu, D. O. Li, C. Gong, J. Warner, Q. H. Wang, D. Blankschtein, M. S. Strano, J. Phys. Chem. C 2017, 121, 14312

[22] C. Sun, M. S. H. Boutilier, H. Au, P. Poesio, B. Bai, R. Karnik, N. G. Hadjiconstantinou, Langmuir 2014, 30, 675.

[23] M. Shan, Q. Xue, N. Jing, C. Ling, T. Zhang, Z. Yan, J. Zheng, Nanoscale 2012, 4, 5477.

[24] H. Du, J. Li, J. Zhang, G. Su, X. Li, Y. Zhao, J. Phys. Chem. C 2011, 115, 23261.

[25] Y. Tao, Q. Xue, Z. Liu, M. Shan, C. Ling, T. Wu, X. Li, ACS Appl. Mater. Interfaces 2014, 6 , 8048 .

[26] S. Choubak, P. L. Levesque, E. Gaufres, M. Biron, P. Desjardins, R. Martel, J. Phys. Chem. C 2014, 118, 21532.

[27] Y. Zhang, Z. Li, P. Kim, L. Zhang, C. Zhou, ACS Nano 2012, 6, 126

[28] S. Bae, H. R. Kim, Y. Lee, X. Xu, J.-S. Park, Y. Zheng, J. Balakrishnan, T. Lei, H. Ri Kim, Y. Il Song, Y.-J. Kim, K. S. Kim, B. Ozyilmaz, J.H. Ahn, B. H. Hong, S. Iijima, H. R. Kim, Y. Il Song, Y.-J. Kim, K. S. Kim, B. Ozyilmaz, J.-H. Ahn, B. H. Hong, S. Iijima, Nat. Nanotechnol. 2010, 5,574

[29] E. S. Polsen, D. Q. McNerny, B. Viswanath, S. W. Pattinson, A. J. Hart, Sci. Rep. 2015, 5, 10257.
[30] M. Eddaoudi, J. Kim, N. Rosi, D. Vodak, J. Wachter, M. O'Keeffe, O. M. Yaghi, Science 2002, 295, 469.

[31] K. Li, D. H. Olson, J. Seidel, T. J. Emge, H. Gong, H. Zeng, J. Li, J. Am. Chem. Soc. 2009 131, 10368.

[32] Y. Peng, Y. Li, Y. Ban, H. Jin, W. Jiao, X. Liu, W. Yang, Science 2014, 346, 1356

[33] G. He, M. Dakhchoune, J. Zhao, S. Huang, K. V. Agrawal, Adv. Funct. Mater. 2018, DOI: 10.1002/adfm.201707427.

[34] J. Cejka, A. Corma, S. Zones, 'Zeolite and Catalysis: Synthesis, Reactions and Applications', Wiley, 2010.

[35] W. J. Roth, P. Nachtigall, R. E. Morris, P. S Wheatley, V. R. Seymour, S. E. Ashbrook, P. Chlubná, L. Grajciar, M. Položij, A. Zukal, O. Shvets, J. Cejka, Nat. Chem. 2013, 5, 628.

[36] International Zeolite Association, http://europe. iza-structure.org/IZA-SC/ftc table.php.

[37] A. I. Lupulescu, J. D. Rimer, Science 2014, 344 , 729.

[38] M. Tsapatsis, AIChE J. 2014, 60, 2374.

[39] K. Varoon, X. Zhang, B. Elyassi, D. D. Brewer, M. Gettel, S. Kumar, J. a. Lee, S. Maheshwari, A. Mittal, C.-Y. Sung, M. Cococcioni, L. F. Francis, a. V. McCormick, K. a. Mkhoyan, M. Tsapatsis, Science 2011, 334, 72.

[40] V. V Narkhede, H. Gies, Chem. Mater. 2009, 21 4339.

[41] M. Choi, K. Na, J. Kim, Y. Sakamoto, O. Terasaki, R. Ryoo, Nature 2009, 461, 246.

[42] V. Nicolosi, M. Chhowalla, M. G. Kanatzidis, M. S. Strano, J. N. Coleman, Science 2013, 340, 1226419.

[43] A. Corma, V. Fornes, S. B. Pergher, T. L. M Maesen, J. G. Buglass, Nature 1998, 396, 353.

[44] N. Rangnekar, M. Shete, K. V. Agrawal, B. Topuz, P. Kumar, Q. Guo, I. Ismail, A. Alyoubi, S. Basahel, K. Narasimharao, C. W. Macosko, K. A. Mkhoyan, S. Al-Thabaiti, B. Stottrup, M. Tsapatsis, Angew. Chemie Int. Ed. 2015, 54, 6571.

[45] K. V. Agrawal, B. Topuz, T. C. T. Pham, T. H Nguyen, N. Sauer, N. Rangnekar, H. Zhang, K. Narasimharao, S. N. Basahel, L. F. Francis, C. W. Macosko, S. Al-Thabaiti, M. Tsapatsis, K. B. Yoon, Adv. Mater. 2015, 27, 3243.

[46] P. Kumar, K. V. Agrawal, M. Tsapatsis, K. A. Mkhoyan, Nat. Commun. 2015, 6, 7128.

[47] U. Oberhagemann, P. Bayat, B. Marler, H. Gies, J. Rius, Angew. Chemie Int. Ed. 1996, 35, 2869.

[48] E. Wirnhier, M. Döblinger, D. Gunzelmann, J. Senker, B. V. Lotsch, W. Schnick, Chem. - A Eur. J. 2011, 17, 3213.

[49] J. Mahmood, E. K. Lee, M. Jung, D. Shin, I.-Y Jeon, S.-M. Jung, H.-J. Choi, J.-M. Seo, S.-Y. Bae, S.-D. Sohn, N. Park, J. H. Oh, H.-J. Shin, J.-B. Baek, Nat. Commun. 2015, 6, 6486. 